\title{
ON THE PERFORMANCE OF UWB-WBAN MODEM
}

\author{
MOHANAD F. ABDULHAMID ${ }^{1}$, ONYANGO BEN SEWE ${ }^{2}$ \\ ${ }^{1}$ AL-Hikma University, Iraq, ${ }^{2}$ University of Nairobi, Kenya \\ E-mail: moh1hamid@yahoo.com, researcher12018@yahoo.com
}

\begin{abstract}
Wireless Body Area Networks (WBANs) have been developed as the human-body monitoring systems to predict, diagnose, and treat diseases. Since the signal transmission in WBANs takes place in or around the human body the channel fading significantly affects packet error rate and overall network performance. This paper focuses on the design and demonstration of an ultra-wideband (UWB) modem to be used in the WBAN applications, and the evaluation of its performance over Rayleigh fading channel. Results show how the fading channel affects the performance of the system.
\end{abstract}

Keywords: UWB modem, WBAN applications

\section{INTRODUCTION}

The advancement in technology can also be seen in miniaturization of electronic devices, sensors, battery and wireless communication, which have led to the development of WBAN. WBAN in simple terminology, can be described as a network around the body which consist of smart miniaturized devices that are able to sense, process and communicate [1].

Typical body area network kits consist of battery, sensor, signal processor, and a transceiver (modem) [1]. A modem is a device that modulates an analog signal, encode it then transmit it, at the same time, the modem receives the signal, decodes it and demodulate it. The main objective of a transceiver is to produce a signal that can be transmitted easily and then decoded to produce the original signal.

The applications of WBAN include healthcare applications, military applications, lifestyle and sports, and monitoring of persons operating in harsh or hostile environments.

Healthcare applications are typically associated with low data rates needed to communicate vital data about human health e.g. heart rate, brain activity, blood pressure, muscle activity, blood sugar level, body temperature, levels of oxygen in the blood, motion etc. the BAN allows reliable monitoring and data transfer for patients without interfering with their mobility $[1,2,3]$.

In the military applications, a battle dress uniform is integrated with a BAN that connects devices such as life support sensors, cameras, and health monitoring global positioning system (GPS). These devices relay real time information. Future advancements will include missile detection sensors and this will indeed revolutionize warfare [1, 2, 3].

Lifestyle and sports are revolutionized since new services like wearable entertainment systems, navigation support in the car or while walking, museum or city guide, heart rate and performance monitoring in sports using muscle activity sensors are made possible by the BAN technology.

In monitoring of persons operating in harsh or hostile environments, there are professions or jobs that require the integration of BAN, for example, miners, fire fighters, etc. to monitor their health and also improve the general working conditions.

Because of the rather simple hardware realizations and the energy efficiency, UWB communication has become one promising technology for the use in WBAN. UWB technology provides the high rate of data transmission due to its relatively large bandwidth of transmission. UWB spans a frequency range of $3.1 \mathrm{GHz}$ to $10.6 \mathrm{GHz}$ with a transmission bandwidth of more than $20 \%$ of its center frequency i.e. more than $500 \mathrm{MHz}$. Based on this transmission bandwidth, it can be seen that the white Gaussian channel capacity of a UWB system is large for a given SNR according to Hartley Shannon law. Some works related to the use of UWB system in WBAN applications are found in $[4,5]$.

\section{EWB MODEM DESIGN}

Since the WBAN sensors have an integrated signal processing chips, the input to the transceiver is in digital form hence no need to include source coding as part of the transceiver design. The physical UWB transceiver design simulation which is done in Matlab simulink includes:

1. Random binary generator

2. Concatenated codes

3. QPSK modulator/ demodulator

4. OFDM transceiver.

5. Channel

\subsection{Random binary generator}

The Bernoulli binary generator is used to generate random binary digits using the Bernoulli distribution. It produces a zero bit (0) with a probability of $p$ and a one bit (1) with probability of $1-p$. 
In this case an equiprobable situation is simulated where both ' 0 ' and ' 1 ' are produced with a probability of 0.5 . The output of this generator is frame based having 256 bits per frame at a sampling rate of $1 / 528 \mathrm{MHz}$.

\subsection{Concatenated codes}

In wireless communications, burst errors occur due to the reflection of the symbols on large surfaces e.g. buildings, trees, hills etc. in addition, random errors also occur due to the thermal noise generated in the electronic circuitry. This calls for a coding scheme with a large codeword length. A serial concatenation of codes is the most commonly used for power - limited systems.

In this case, a $(48,32,8)$ Reed-Solomon (R-S) code (outer code) with symbols over GF $\left(2^{8}\right)$ and a $(2,1)$ convolution code of constraint length 7 was used.

\subsubsection{Reed Solomon coding/decoding}

A $(48,32,8)$ R-S code over GF $\left(2^{8}\right)$ was obtained by code shortening scheme of puncturing (zero padding) as shown in Figure 1 in a matlab simulink model. This code corrects up to 8 symbol errors out of the 48 symbols.

Since R-S encoder is a non-binary coding scheme, the 256 bit frame from the Bernoulli generator is converted to integers using bit to integer converter of 8 , resulting into 32 bytes which is the input sequence to the R-S encoder subsystem.

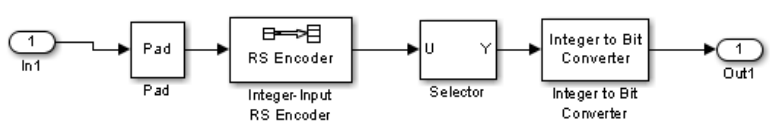

Figure 1. R-S encoder

The 32 byte sequence is zero padded to 239 message bytes which is then fed to the integer input R-S encoder. This block adds 16 parity check bytes to give 255 codeword length. Since we are interested in the 48 code words, the zero padded 255 code words is passed through a selector to give the 48 codewords hence a (48, $32,8) \mathrm{R}-\mathrm{S}$ code achieved from the $(255,239,8) \mathrm{R}-\mathrm{S}$ code. The 48 bytes is converted back to binary to give 384 bits which is passed through to the convolution encoder.

In the decoder shown in Figure 2, the 384 bits is converted to bytes, zero padded to and fed to the decoder which decodes the message i.e. corrects any error introduced during the transmission and removes the parity check bits. The zero padded 239 message digits from the decoder is passed through a selector to obtain the 32 original message digits which are then converted back to binary.

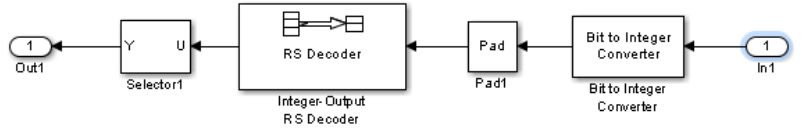

Figure 2. R-S decoder

\subsubsection{Convolution coding/Viterbi decoding}

This convolution code has an information rate of $1 / 2$ and constraint length of 7 .It uses the polytrellis (7, [171 133]) function to create a trellis using the constraint length, code generator (octal) and feedback connection (octal). As can be seen from the Figure 3,

$$
\text { Output }[\mathrm{a}, \mathrm{b}]=\operatorname{input}\left[x_{1}, x_{2}\right]
$$

Where $\quad \mathrm{x}_{1}=(1111001)=(171)_{8}$ $\mathrm{x}_{2}=(1011011)=(133)_{8}$

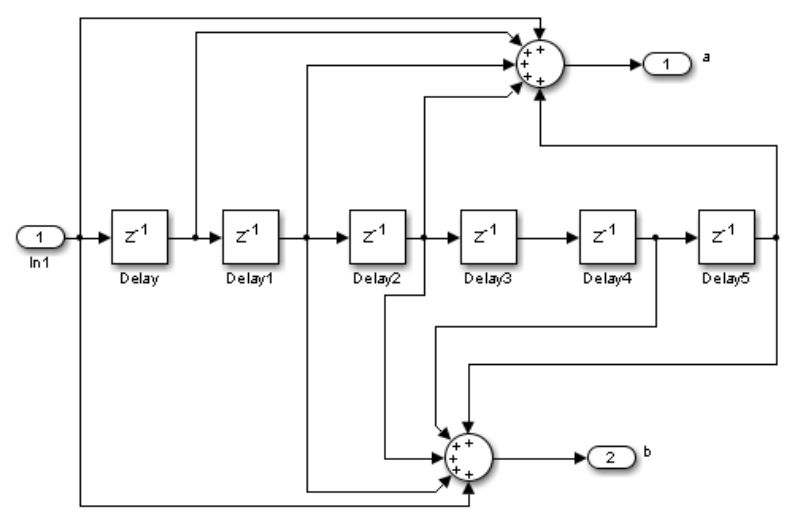

Figure 3. Polytrellis $(7,[171,133])$ structure

The Viterbi decoder also uses the same polytrellis function while decoding the information transmitted. Since the information rate is $1 / 2$, this implies that for every one bit, two codewords are produced hence the output of the convolution encoder is 768 bits. The Viterbi decoder, detects and corrects the random errors and removes the parity check bits hence its output is 384 bits.

\subsection{QPSK modulator/demodulator}

The quadrature phase shift keying(QPSK) modulator maps the binary digits from the information sequence into discrete phases of the carrier as shown in Figure 4.

The 768 message bits are converted to integers and then fed into the QPSK modulator which maps the 384 integers to complex 384 integers. QPSK demodulator performs inverse operation of QPSK modulator. 


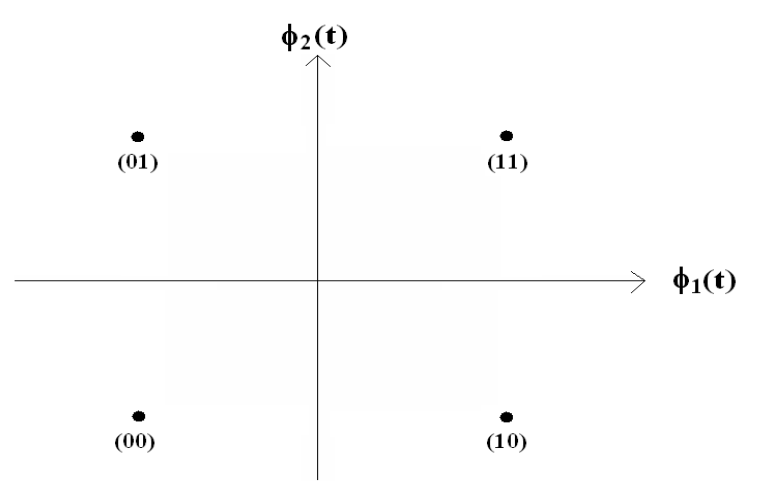

Figure 4. QPSK constellation mapping

\subsection{OFDM transceiver}

Orthogonal frequency division multiplexing (OFDM) symbol consist of the data carriers, guard subcarriers and the cyclic prefix, with time durations as shown in Figure 5. In this design, 128 subcarriers are used, with 96 being data carriers, 12 pilots and 20 nulls for guard. A cyclic prefix of 32 subcarriers is appended.

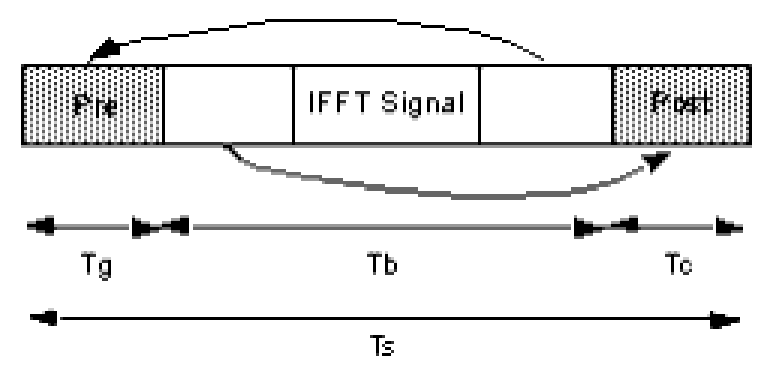

Figure 5. OFDM symbol

The 384 complex integers are rearranged to form $96 \times 4$ array matrix. The matrix is regrouped as:

$$
\{1,[2: 10],[11: 19],[20: 28],[29: 37], \text { [38:46], [47:50], }
$$$$
\text { [51:54], [55:62], [63:70], [71:78], [79:86], [87:96]\} }
$$

to allow the insertion of the pilots. The pilots are inserted at the positions $(2,12,22,32,42,52,61,70,79,88,97$, 108).

The guards are then inserted at the beginning and end of the data carriers. The symbol is then passed through the Inverse Fast Fourier Transform (IFFT) to create the orthogonal signals.

A cyclic prefix is appended by rearranging and reordering the sequence as [97:128 1:128]. This command repeats the last 32 carriers at the beginning of the OFDM symbol.

The OFDM symbol is then power scaled and transmitted via the channel. The OFDM transmitter is designed as shown in Figure 6.
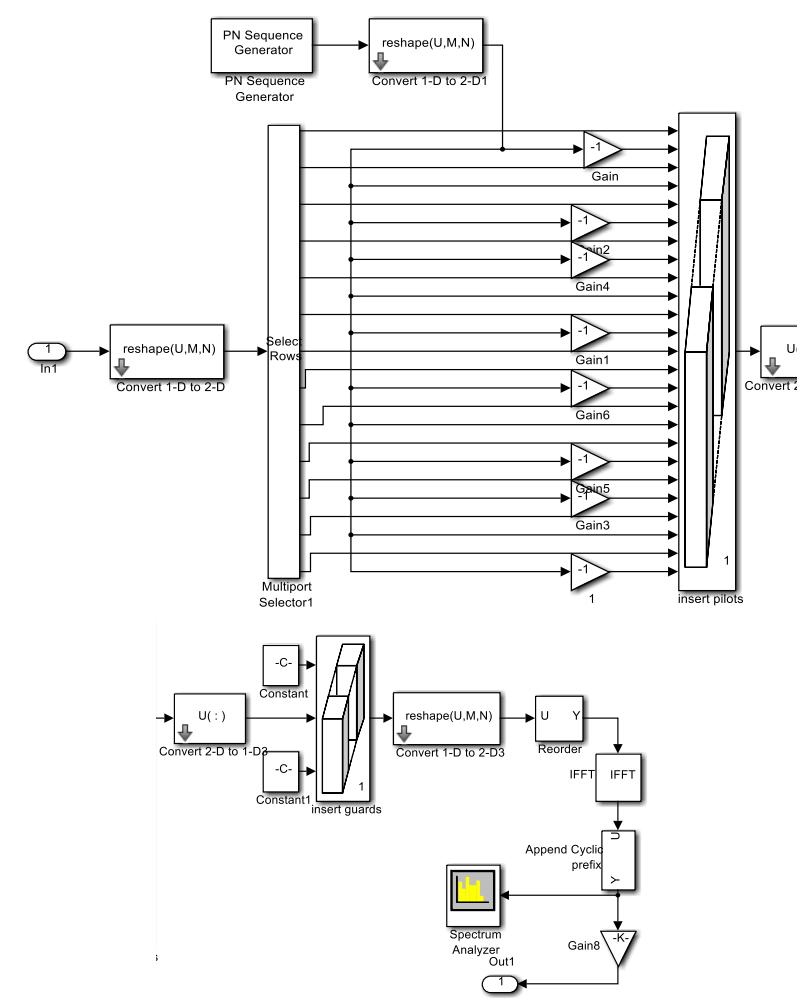

Figure 6. OFDM transmitter

At the OFDM receiver shown in Figure 7, the received symbol is down scaled and the cyclic prefix is removed by selecting the message portion. The received message is then transformed by Fast Fourier Transform (FTT) to remove the orthogonality. The guards are then removed and subsequently the pilots. The remaining data stream is then rearranged back to the 384 constellation points and then demodulated using QPSK demodulator.
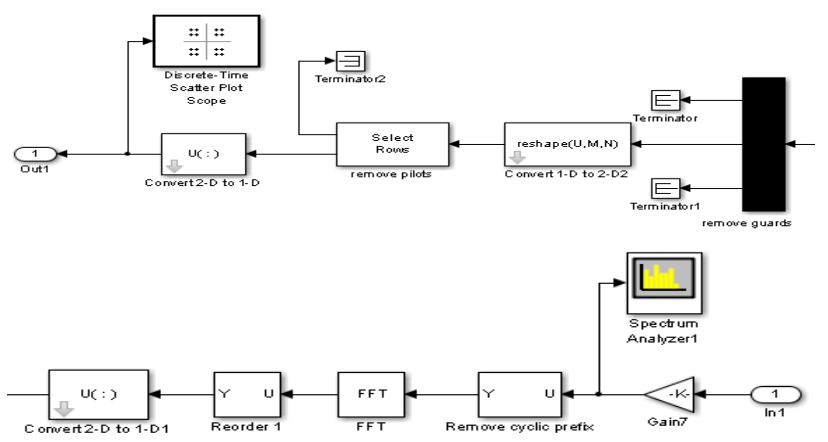

Figure 7. OFDM receiver

\subsection{The channel}

The Rayleigh fading channel depicts a situation where the transmitted signal arrives at the receiver via different paths. This is caused by reflections from surfaces like walls. The different paths have their associated path delays and losses. This channel multiplies the input signal by samples of a Rayleigh-distributed complex random process. In this design up to three paths are simulated and the results displayed. 


\subsection{Overall design diagram}

The overall design is shown in Figure 8.

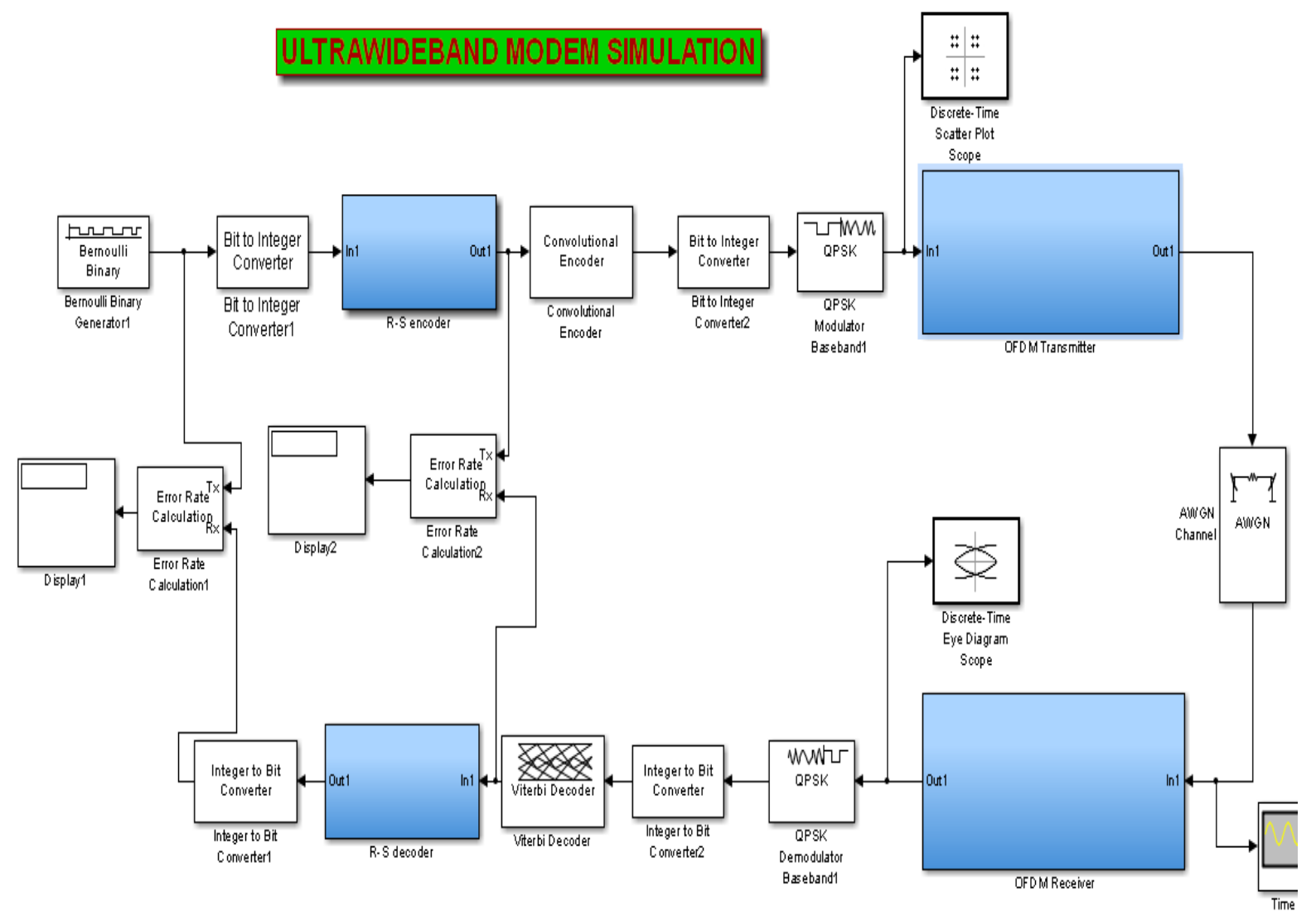

Figure 8. Overall Design

\section{SIMULATION RESULTS}

\subsection{Single path with no delay period}

This case represents ideal channel due to single path, so there is no intersymbol interference, and no noise interference, so the transmitted signal (Figure 9) and received signal (Figure 10) are identical, and the error rate calculation is zero as shown in Figure 11.

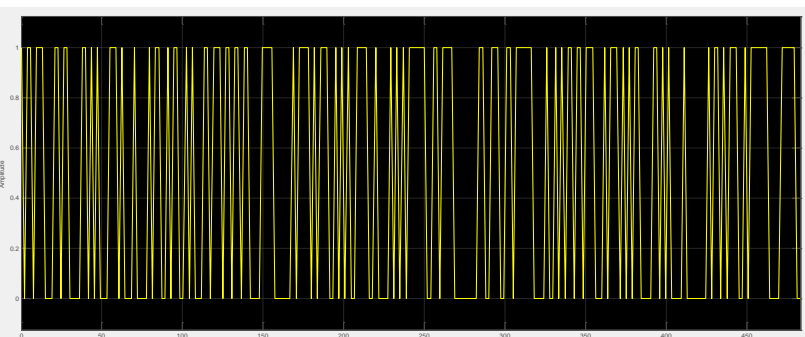

Figure 9. Transmitted signal

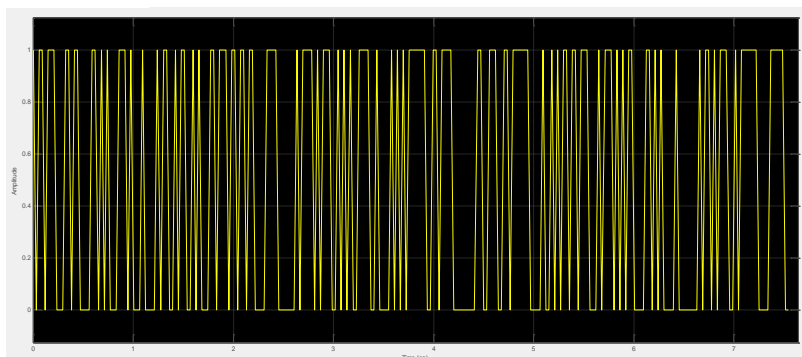

Figure 10. Received signal

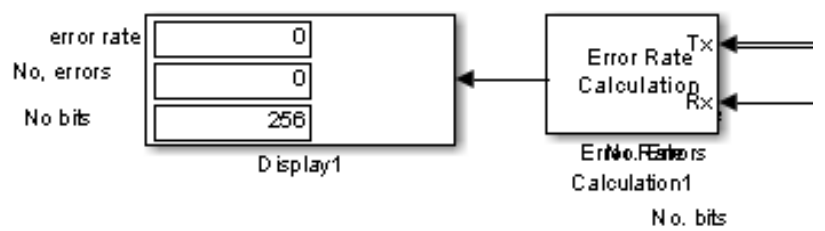

Figure 11. Error rate calculation

From the signal spectrum shown in Figure 12, it is noted that the transmitted signal has a power spectral density (PSD) of around $-80 \mathrm{dbm} / \mathrm{Hz}$. Thus this modem ensures low spectral density transmission required by the regulating bodies. 


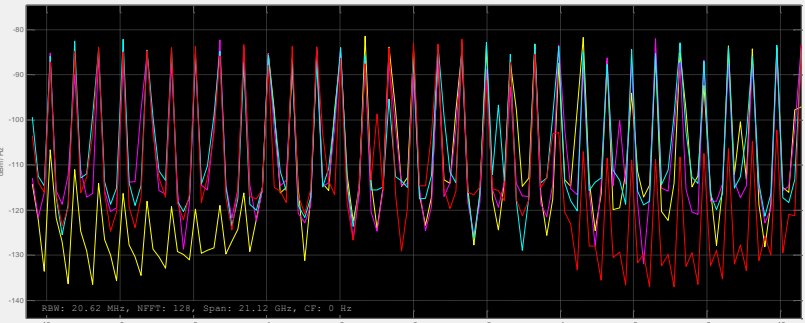

Figure 12. Signal spectrum

Also, from eye diagram shown in Figure 13, it can be concluded that a wider eye, a lower interference. So, the signal is interference free in this case as the eye is wide.
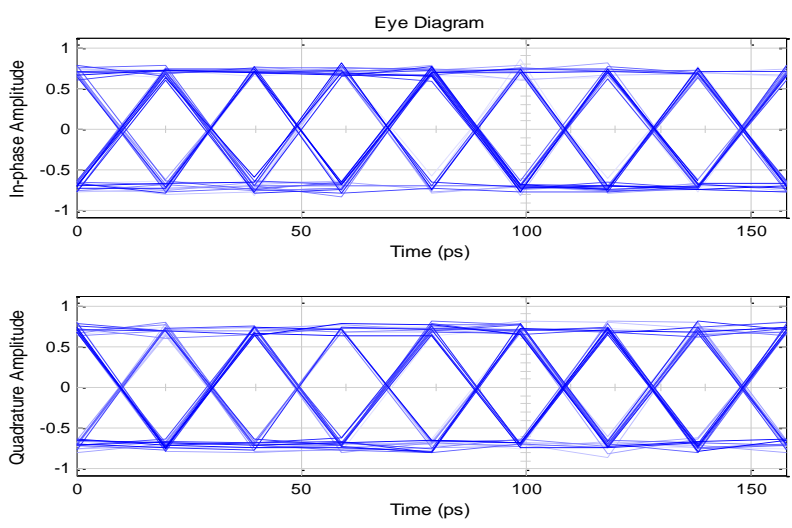

Figure 13. Eye diagram

\subsection{Multiple paths with no delay period}

The transmitted signal (Figure 14) and received signal (Figure 15) are not identical due to intersymbol interference caused by multipath propagation, and the error rate calculation is 0.488 as shown in Figure 16 . Also, Figure 17 shows the signal spectrum, while Figure 18 shows the eye diagram which shows the distortion due to interference.

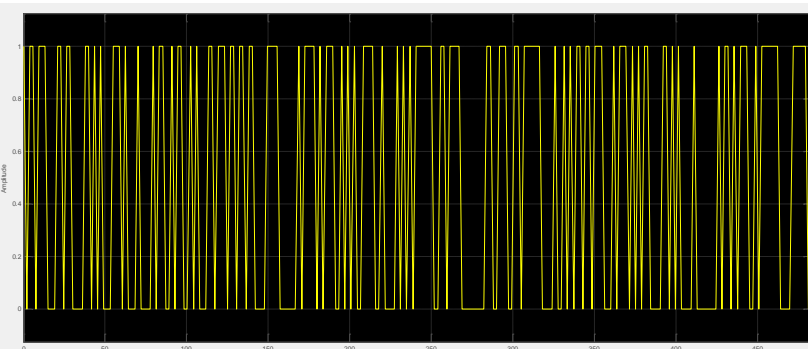

Figure 14. Transmitted signal

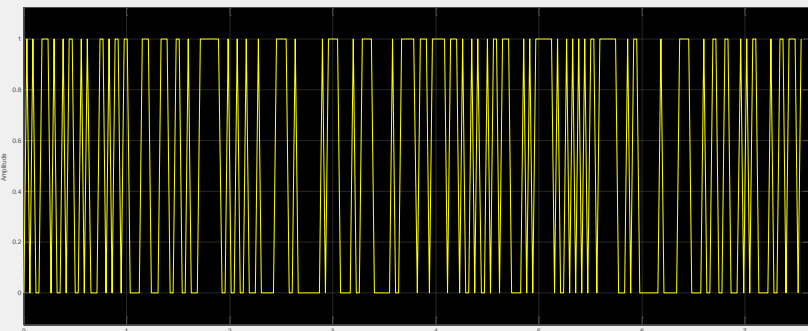

Figure 15. Received signal

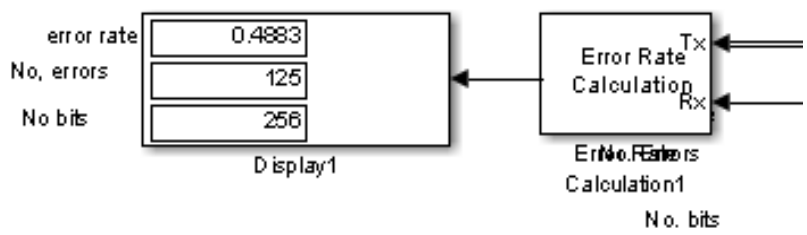

Figure 16. Error rate calculation

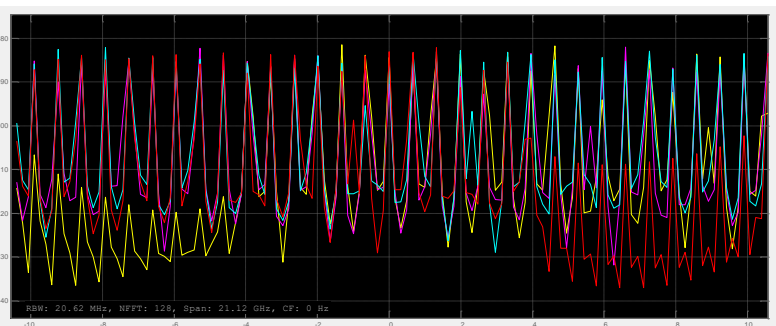

Figure 17. Signal spectrum
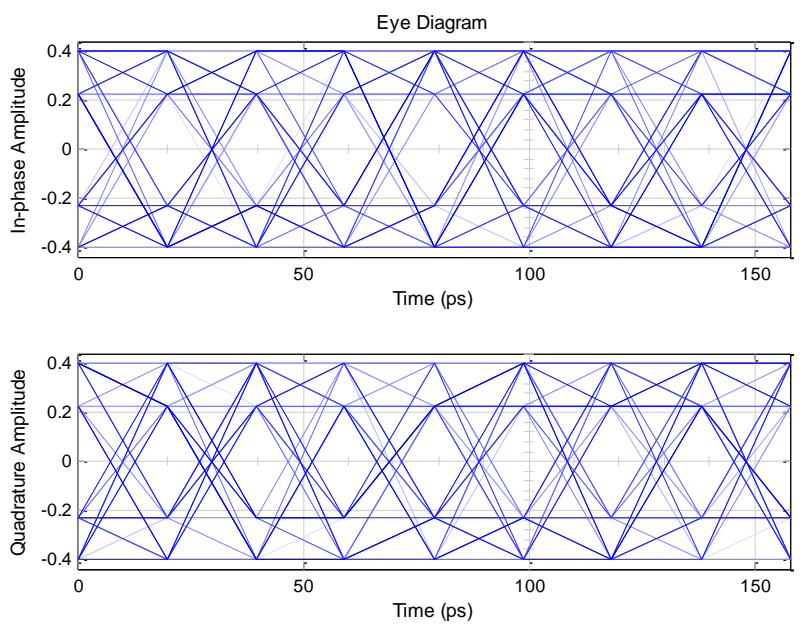

Figure 18. Eye diagram

\section{CONCLUSION}

This paper studied both the UWB wireless communication systems and WBAN applications and then designed and demonstrated a modem to be used in those applications. The performance of the modem was studied over Rayleigh fading channel. The results showed the effect of multipath propagation. Also, the modem can achieve an error free transmission at a lower power spectral density and at a very high data rate.

\section{REFERENCES}

[1] G. Crosby, et. al., "Wireless body area networks for healthcare: A survey", International Journal of Ad hoc, Sensor \& Ubiquitous Computing, Vol.3, No.3, 2012.

[2] M. Samaneh, et. al., "Wireless body area networks: A survey", IEEE Communications Surveys and Tutorials, Vol.16, Issue 3, 2014.

[3] G. Ragesh, and K. Baskaran, "An overview of applications, standards and challenges in futuristic wireless body area networks", International Journal 
of Computer Science Issues, Vol. 9, Issue 1, No. 2, 2012.

[4] E. Hamza, and R. Majeed, "MAC Protocol for UWB wireless body area networks", American Scientific Research Journal for Engineering, Technology, and Sciences, Vol. 38, No.1, 2017.

[5] M. Ali, "Low power FM-UWB transmitter for wireless body area networks", Ph.D. thesis, Electronics Research Institute, Egypt, 2017.

[6] O. Ben Sewe, "Ultra-wideband modem for wireless body area network applications", Graduation Project, Nairobi University, Kenya, 2014. 